ORIGINAL RESEARCH PAPER

\section{BAYESIAN ESTIMATION AND AN APPLICATION OF GIBBS SAMPLING TECHNIQUE AND RANDOIM WALK METROPOLIS - HASTING ALGORITHMI IN TWO PHASE LINEAR REGRESSION MODEL}

\section{Statistics}

KEY WORDS: Gibbs Sampling and RWM-H (Random Walk Metropolis - Hasting) Algorithm, Two Phase Linear Regression (TPLR) Model, Bayesian Estimation, Change Point.
Dr. Maitreya N. Acharya
Assistant Professor, Department of Statistics, School of Sciences, Gujarat University, Ahmedabad, Gujarat, India.

Here, in this research paper, we have applied the Gibbs Sampling Technique and RWM-H (Random Walk Metropolis Hasting) Algorithm for the Bayesian Estimation of $\mathrm{m}, \beta 1, \beta 2$ and 1/2. Also we have assumed that at some point of time say ' $m$ ', the co-efficient of regression changes from $\beta 1$ to $\beta 2$. Further, we have discussed about the effects of prior information on the Bayes estimates on the basis of the TPLR (Two Phase Linear Regression) Model with a Bayesian approach.

\title{
INTRODUCTION:
}

The regression coefficients are assumed constant as far as regression analysis is concerned, in general practice. A model can be suggested on the basis of the theoretical or empirical deliberations as far as real life situations are concerned. Here, we note that the model changes occasionally in either one or more of its parameters, most of the times. Generally, we all know that regression analysis is widely applicable in the fields of social, medical and engineering science. Here, we have emphasized on the point where the unknown change occurred and so we have focused on the shift point parameter. It is so because regression analysis indexes when or where the unknown change occurred in the system.

The Gibbs sampling technique for generating random variables from a marginal distribution is applied here in an indirect manner. Further, we note that we are not calculating the density. We have taken an assumption that we have a joint density as $\boldsymbol{f}\left(\boldsymbol{x}, \boldsymbol{y}_{1}, \ldots, \boldsymbol{y}_{\boldsymbol{p}}\right)$ and we want to obtain characteristic values of the marginal density

$$
f(x)=\int \ldots \int f\left(x, y_{1}, \ldots, y_{p}\right) d y_{1} \ldots d y_{p}
$$

It may be mean or variance. In general, it may be considered as the most natural and straightforward approach to calculate $\boldsymbol{f}(\boldsymbol{x})$ and use it to obtain the desired characteristic values. There are many cases where the analytical and numerical integration in (1) are very much difficult to perform in practice. Therefore, we have applied Gibbs sampler in such cases as it provides an alternative method to obtain $\boldsymbol{f}(\boldsymbol{x})$.

On the basis of some research done in the past, we have assumed that we already have some technical knowledge about the parameter of the models which are already available. We have taken some notable references of researchers such as Arnold Zellner (1971), A.F.M. Smith (1980) and A.K. Bansal and S. Chakravarty (1996) who have studied the Bayesian estimation for the regression coefficient and change point of the TPLR model.

Now-a-days, simulation has become an increasingly important technique as an alternative to numerical as well as analytical approximation techniques. In this paper, we have shown how the routine Bayesian Analysis of the Two Phase Linear Regression (TPLR) model is made possible using simulation methods based on Markov Chain Monte Carlo (MCMC) technique. In the last decade, there has been a significant growth of interest in MCMC methods which include further refinements of standard Monte Carlo sampling techniques. We can say so very clearly from the developments which have taken place in the last decade.

It was the joint research work of Gelfand and Smith (1990) that gave the realization that Markov Chain could be used in wide variety of situations through their research studies and conclusions. Bayesian Analysis for the Block and Basu Bivariate Exponential distribution was done by Jorge A. Achcar and Roseli A. Leandro (1998) using Metropolis Algorithm with Gibbs steps and sampling technique. S.K. Upadhyay and N. Vasistha (2000) have worked jointly to overcome the computational difficulties using simulation approaches to Bayesian computation in reliability models, using MCMC methods. The understanding of how the development of Monte Carlo Methodology did not change our solution to the problems, but it changed the way of our thinking about the problems was given by Christian Robert and George Casella (2011).

\section{TWO PHASE LINEAR REGRESSION MODEL:}

We shall consider the following Two Phase Linear Regression (TPLR) Model for our study.

$Y_{t}=\left\{\begin{array}{rr}\beta_{1} X_{t}+\epsilon_{t} & t=1,2, \ldots, m \\ \beta_{2} X_{t}+\epsilon_{t} & t=m+1, m+2, \ldots, n\end{array}\right.$

Here, $\boldsymbol{X}_{\boldsymbol{t}}$ is a non-stochastic explanatory variable. $\boldsymbol{\beta}_{1}$ and $\boldsymbol{\beta}_{2}$ are the regression parameters where $\boldsymbol{\beta}_{1} \neq \boldsymbol{\beta}_{2}$. The independently and identically distributed random errors are shown as $\epsilon_{t}$ which follow the Normal distribution, i.e. $\boldsymbol{N}\left(\mathbf{0}, \sigma^{2}\right)$. Further, we note that variance is strictly positive, i.e. $\left(\sigma^{2}>0\right)$

\section{BAYES ESTIMATION:}

The likelihood function of $\boldsymbol{\beta}_{1}, \boldsymbol{\beta}_{2}, \sigma^{-2}$ and ' $\boldsymbol{m}$ ' on the basis of the sample information $\boldsymbol{Z}=\left(\boldsymbol{x}_{t}, \boldsymbol{y}_{t}\right)$, where $t=1,2, \ldots, \boldsymbol{m}, \boldsymbol{m}+\mathbf{1}, \ldots, \boldsymbol{n}$ is taken as under:

$$
L\left(\beta_{1}, \beta_{2}, \sigma^{-2}, m \mid Z\right)=\frac{1}{(2 \pi)^{\frac{n}{2}}}\left(e^{-\frac{A}{2 \sigma^{2}}}\right) \cdot\left(\sigma^{-n}\right) \cdot \exp \left[-\frac{1}{2} \beta_{1}^{2}\left(\frac{S_{m 1}}{\sigma^{2}}\right)+\beta_{1}\left(\frac{S_{m 3}}{\sigma^{2}}\right)\right] \cdot \exp \left[\frac{-1}{2} \beta_{2}^{2}\left(\frac{S_{n 1}-S_{m 1}}{\sigma^{2}}\right)+\beta_{2}\left(\frac{S_{m 4}}{\sigma^{2}}\right)\right](3)
$$

where,

$$
\begin{array}{ll}
S_{k 1}=\sum_{\mathrm{i}=1}^{\mathrm{k}} \mathrm{x}_{\mathrm{i}}^{2}, & S_{k 2}=\sum_{\mathrm{i}=1}^{\mathrm{k}} \mathrm{x}_{\mathrm{i}} \mathrm{y}_{\mathrm{i}}, \\
S_{m 3}=S_{m 2}, & S_{m 4}=S_{n 2}-S_{m 2}
\end{array}
$$$$
A=\sum_{i=1}^{n} y_{i}^{2}
$$

APPLICATION OF GAMMA PRIOR ON $1 / \sigma^{2}$ AND INFORMATIVE PRIORS ON $\beta_{1}$ AND $\beta_{2}$ WITH UNKNOWN $\sigma^{-2}$ :

Here, the TPLR model (2) is taken into consideration with unknown $\sigma^{-2}$ just as it was used by Broemeling et.al. (1987). Further, uniform prior for change point ' $m$ ' is assumed as $g(m)=\frac{1}{n-1}$ 
Moreover, we have normal prior density on $\beta_{1}$ and $\beta_{2}$ as,

$$
\begin{aligned}
& g\left(\beta_{1}\right)=\frac{1}{\sqrt{2 \pi} \sigma_{1}} e^{-\frac{1}{2}\left(\frac{\beta_{1}-\mu_{1}}{\sigma_{1}}\right)^{2}} \\
& g\left(\beta_{2}\right)=\frac{1}{\sqrt{2 \pi} \sigma_{2}} e^{-\frac{1}{2}\left(\frac{\beta_{2}-\mu_{2}}{\sigma_{2}}\right)^{2}}
\end{aligned}
$$

Further, we assume the $\operatorname{Gamma}(\boldsymbol{c}, \boldsymbol{d})$ prior for $\frac{1}{\sigma^{2}}$ as under:

$$
g\left(\frac{1}{\sigma^{2}}\right)=\frac{c^{d}}{\Gamma d}\left(\frac{1}{\sigma^{2}}\right)^{d-1} e^{-\frac{c}{\sigma^{2}}} \quad \sigma^{2}>0
$$

Also we note that $\boldsymbol{c}$ and $\boldsymbol{d}$ are the hyper parameter which can be obtained by using following relations:

$d=\frac{1}{\emptyset^{2}}, \quad c=\frac{1}{\mu_{3} \emptyset^{2}}$.

We note that $\mu_{3}$ and $\varnothing$ are prior mean and prior coefficient of variation of $\frac{1}{\sigma^{2}}$ respectively.

Hence, joint prior p.d.f. of $\boldsymbol{\beta}_{1}, \boldsymbol{\beta}_{2}, \boldsymbol{\sigma}^{-2}$ and ' $\boldsymbol{m}$ ' will be:

$g\left(\beta_{1}, \beta_{2}, \sigma^{-2}, m\right)=\frac{c^{d}}{2 \pi \sigma_{1} \sigma_{2} \sigma^{2}(d-1) \Gamma d(n-1)} e^{-\frac{1}{2}\left(\frac{\beta_{1}-\mu_{1}}{\sigma_{1}}\right)^{2}} e^{-\frac{1}{2}\left(\frac{\beta_{2}-\mu_{2}}{\sigma_{2}}\right)^{2}}$

Using Likelihood function (3) with the joint prior density, the joint posterior density of $\beta_{1}, \beta_{2}, \sigma^{-2}, m$ say $g\left(\beta_{1}, \beta_{2}, \sigma^{-2}, m \mid Z\right)$ will be:

$g\left(\beta_{1}, \beta_{2}, \sigma^{-2}, m \mid Z\right)=K_{1}\left[e^{\left[-\frac{1}{2} \beta_{1}{ }^{2} B_{1}+\beta_{1} A_{1}\right]} e^{\left[-\frac{1}{2} \beta_{2}{ }^{2} B_{2}+\beta_{2} A_{2}\right]} e^{-\frac{1}{\sigma^{2}}\left(\frac{A+B}{2}+c\right)} \sigma^{-2\left(\frac{n}{2}+d-1\right)}\right] / h_{1}(z)$

where,

$K_{1}=\frac{c^{d} \exp \left[-\frac{1}{2}\left(\left(\frac{\mu_{1}}{\sigma_{1}}\right)^{2}+\left(\frac{\mu_{2}}{\sigma_{2}}\right)^{2}\right)\right]}{(2 \pi)^{n / 2} 2 \pi \Gamma d(n-1)}$

$A_{1}=\frac{\mu_{1}}{\sigma_{1}^{2}}+\frac{S_{m 3}}{\sigma^{2}} \quad B_{1}=\frac{1}{\sigma_{1}^{2}}+\frac{S_{m 1}}{\sigma^{2}}$

$A_{2}=\frac{\mu_{2}}{\sigma_{2}{ }^{2}}+\frac{S_{m 4}}{\sigma^{2}} \quad B_{2}=\frac{1}{\sigma_{2}{ }^{2}}+\frac{S_{n 1}-S_{m 1}}{\sigma^{2}}$

$A=\sum_{i=1}^{n} y_{i}^{2} \quad B=\beta_{1}{ }^{2} S_{m 1}+2 \beta_{1} S_{m 3}+\beta_{2}{ }^{2}\left(S_{n 1}-S_{m 1}\right)+2 \beta_{2} S_{m 4}$

$h_{1}(Z)$ is the marginal density of $z$ given by,

$$
\begin{aligned}
& \boldsymbol{h}_{\mathbf{1}}(\boldsymbol{Z})=\sum_{m=1}^{n-1} \int_{0}^{\infty} \int_{-\infty}^{\infty} \int_{-\infty}^{\infty} L\left(\beta_{1}, \beta_{2}, \sigma^{-2}, m \mid Z\right) \cdot g\left(\beta_{1}, \beta_{2}, \sigma^{-2}, m\right) d \beta_{1} d \beta_{2} d \sigma^{-2} \\
& =\sum_{m=1}^{n-1} \int_{-\infty}^{\infty} e^{\left[-\frac{1}{2} \beta_{1}{ }^{2} B_{1}+\beta_{1} A_{1}\right]} d \beta_{1} \int_{-\infty}^{\infty} e^{\left[-\frac{1}{2} \beta_{2}{ }^{2} B_{2}+\beta_{2} A_{2}\right]} d \beta_{2} \int_{0}^{\infty} e^{-\frac{1}{\sigma^{2}}\left(\frac{A+B}{2}+c\right)} \sigma^{-2\left(\frac{n}{2}+d-1\right)} d \sigma^{-2}
\end{aligned}
$$

(8)

Marginal posterior of $\beta_{1}, \beta_{2}, \sigma^{-2}$ and ' $m$ ' respectively will be:

$$
\begin{gathered}
\boldsymbol{g}\left(\boldsymbol{\beta}_{\mathbf{1}} \mid \boldsymbol{Z}\right)=\frac{\left[\sum_{m=1}^{n-1} e^{\left[-\frac{1}{2} \beta_{1}{ }^{2} B_{1}+\beta_{1} A_{1}\right]} \int_{-\infty}^{\infty} e^{\left[-\frac{1}{2} \beta_{2}{ }^{2} B_{2}+\beta_{2} A_{2}\right]} d \beta_{2} \int_{0}^{\infty} e^{-\frac{1}{\sigma^{2}}\left(\frac{A+B}{2}+c\right)} \sigma^{-2\left(\frac{n}{2}+d-1\right)} d \sigma^{-2}\right]}{h_{1}(Z)} \\
\boldsymbol{g}\left(\boldsymbol{\beta}_{2} \mid \boldsymbol{Z}\right)=\frac{\left[\sum_{m=1}^{n-1} e^{\left[-\frac{1}{2} \beta_{2}{ }^{2} B_{2}+\beta_{2} A_{2}\right]} \int_{-\infty}^{\infty} e^{\left[-\frac{1}{2} \beta_{1}{ }^{2} B_{1}+\beta_{1} A_{1}\right]} d \beta_{1} \int_{0}^{\infty} e^{-\frac{1}{\sigma^{2}}\left(\frac{A+B}{2}+c\right)} \sigma^{-2\left(\frac{n}{2}+d-1\right)} d \sigma^{-2}\right]}{h_{1}(Z)} \\
\boldsymbol{g}\left(\boldsymbol{\sigma}^{-2} \mid \boldsymbol{Z}\right)=\frac{\left[\sum_{m=1}^{n-1} e^{-\frac{1}{\sigma^{2}}\left(\frac{A+B}{2}+c\right)} \sigma^{-2\left(\frac{n}{2}+d-1\right)} \int_{-\infty}^{\infty} e^{\left[-\frac{1}{2} \beta_{1}{ }^{2} B_{1}+\beta_{1} A_{1}\right]} d \beta_{1} \int_{-\infty}^{\infty} e^{\left[-\frac{1}{2} \beta_{2}{ }^{2} B_{2}+\beta_{2} A_{2}\right]} d \beta_{2}\right]}{h_{1}(Z)} \\
\boldsymbol{g}(\boldsymbol{m} \mid \boldsymbol{Z})=\frac{\left[\int_{-\infty}^{\infty} e^{\left[-\frac{1}{2} \beta_{1}{ }^{2} B_{1}+\beta_{1} A_{1}\right]} d \beta_{1} \int_{-\infty}^{\infty} e^{\left[-\frac{1}{2} \beta_{2}{ }^{2} B_{2}+\beta_{2} A_{2}\right]} d \beta_{2} \int_{0}^{\infty} e^{-\frac{1}{\sigma^{2}}\left(\frac{A+B}{2}+c\right)} \sigma^{-2\left(\frac{n}{2}+d-1\right)} d \sigma^{-2}\right]}{h_{1}(Z)}
\end{gathered}
$$

Now, the Bayes estimator of any function of parameter $\alpha$, say $g(\alpha)$ under the squared loss function will be:

$$
E_{\alpha \mid z}(g(\alpha \mid Z))=\int_{0}^{\infty} \alpha(g(\alpha \mid Z)) d \alpha
$$

where, $\boldsymbol{g}(\alpha \mid Z)$ is marginal posterior density of $\boldsymbol{\alpha}$. It is little complicated to compute the equation (13) analytically in this case. Therefore, we propose to use Gibbs sampling and MCMC methods to find the Bayes estimators of $\boldsymbol{\beta}_{1}, \boldsymbol{\beta}_{2}, \sigma^{2}$ and ' $\boldsymbol{m}$ '.

\section{ALGORITHM USING GIBBS SAMPLING:}

The Gibbs sampling procedure is implemented and therefore we re-write (9) as full conditional of $\boldsymbol{\beta}_{1}$ by fixing all other parameters, i.e. $\boldsymbol{\beta}_{2}, \boldsymbol{\sigma}^{-2}$ and ' $\boldsymbol{m}$ '. Hence, full conditional density of $\boldsymbol{\beta}_{1}$ given $\boldsymbol{\beta}_{2}, \sigma^{-2}$ and ' $\boldsymbol{m}$ ' will be as follows:

$$
g\left(\beta_{1} \mid \beta_{2}, \sigma^{-2}, m, Z\right) \propto N\left(\frac{A_{1}}{B_{1}},\left(\frac{1}{\sqrt{B_{1}}}\right)^{2}\right)
$$

where $\boldsymbol{A}_{1}$ and $\boldsymbol{B}_{1}$ are given in equation (7).

Further, we also re-write (10) as full conditional density of $\beta_{2}$ and by fixing all other parameters $\beta_{1}, \sigma^{-2}$ and ' $\mathbf{m}$ ', we get the full conditional density of $\boldsymbol{\beta}_{2}$ given $\boldsymbol{\beta}_{1}, \sigma^{-2}$ and ' $\boldsymbol{m}$ ' as follows:

$$
g\left(\beta_{2} \mid \beta_{1}, \sigma^{-2}, m, Z\right) \propto N\left(\frac{A_{2}}{B_{2}},\left(\frac{1}{\sqrt{B_{2}}}\right)^{2}\right)
$$


where $\boldsymbol{A}_{2}$ and $\boldsymbol{B}_{2}$ are given in equation (7).

Now, we re-write (11) as full conditional density of $\sigma^{-2}$ and by fixing parameters $\boldsymbol{\beta}_{1}, \boldsymbol{\beta}_{2}$ and ' $\boldsymbol{m}$ ', we get the full conditional density of $\sigma^{-2}$ given $\boldsymbol{\beta}_{\mathbf{1}}, \boldsymbol{\beta}_{2}$ and ' $\boldsymbol{m}$ ' is as follows,

$$
g\left(\sigma^{-2} \mid \beta_{1}, \beta_{2}, m, Z\right) \propto \operatorname{gamma}\left(\frac{n}{2}+d, \frac{1}{c+\frac{A+B}{2}}\right)
$$

where, values of $\boldsymbol{A}$ and $\boldsymbol{B}$ are given in equation (7)

We apply Gibbs sampling to generate sample from the full conditional density of $\boldsymbol{\beta}_{1}, \boldsymbol{\beta}_{2}$ and $\boldsymbol{\sigma}^{-2}$ given respectively in (14), (15) and (16). In order to estimate the parameters $\boldsymbol{\beta}_{1}, \boldsymbol{\beta}_{2}$ and $\boldsymbol{\sigma}^{-2}$ we use following algorithm:

\section{ALGORITHM:}

Initialize $\boldsymbol{\beta}_{\mathbf{1}}=\boldsymbol{\beta}_{\mathbf{1 0}}, \boldsymbol{\beta}_{\mathbf{2}}=\boldsymbol{\beta}_{\mathbf{2 0}}, \boldsymbol{\sigma}^{-\mathbf{2}}=\boldsymbol{\sigma}_{\mathbf{0}}^{-2}$ and $\boldsymbol{m}=\boldsymbol{m}_{\mathbf{0}}$ then,

STEP 1: $\quad$ Generate $\beta_{1} \sim N\left(\frac{A_{1}}{B_{1}},\left(\frac{1}{\sqrt{B_{1}}}\right)^{2}\right)$, using Gibbs Sampling.

STEP 2: $\quad$ Generate $\boldsymbol{\beta}_{2} \sim N\left(\frac{A_{2}}{B_{2}},\left(\frac{1}{\sqrt{B_{2}}}\right)^{2}\right)$, using Gibbs Sampling

STEP 3: $\quad$ Generate $\sigma^{-2} \sim$ gamma $\left(\frac{n}{2}+d, \frac{1}{c+\frac{A+B}{2}}\right)$, using Gibbs Sampling.

STEP 4: Repeat the above steps.

\section{APPLICATION OF MCMC TECHNIQUES:}

There is no closed form of posterior distribution of change point (12). That is why we need to propose the use of MCMC techniques to generate the samples from the posterior distribution. To implement the MCMC techniques, we re-write (12) as target function of ' $m$ ', by fixing all other parameters i.e. $\boldsymbol{\beta}_{1}, \boldsymbol{\beta}_{2}$, and $\boldsymbol{\sigma}^{-2}$. Hence full target function of ' $\boldsymbol{m}$ ' given $\boldsymbol{\beta}_{1}, \boldsymbol{\beta}_{2}$, and $\boldsymbol{\sigma}^{-2}$ will be as follows:

$$
\begin{gathered}
g\left(m \mid \sigma^{-2}, \beta_{1}, \beta_{2}, Z\right) \propto e^{-\frac{1}{\sigma^{2}}\left(c+\frac{A+B}{2}\right)} \sigma^{-2\left(\frac{n}{2}+d-1\right)} G_{1 m} G_{2 m} \\
G_{1 m}=\int_{-\infty}^{\infty} e^{\left[-\frac{1}{2} \beta_{1}{ }^{2}\left(\frac{S_{m 1}}{\sigma^{2}}+\frac{1}{\sigma_{1}^{2}}\right)+\beta_{1}\left(\frac{S_{m 3}}{\sigma^{2}}\right)\right]} d \beta_{1}=\int_{-\infty}^{\infty} e^{\left[-\frac{1}{2} \beta_{1}{ }^{2} B_{1}+\beta_{1} A_{1}\right]} d \beta_{1}=\frac{e^{\frac{A_{2}{ }^{2}}{2 B_{1}}} \sqrt{2 \pi}}{\sqrt{B_{1}}} \\
G_{2 m}=\int_{-\infty}^{\infty} e^{\left[-\frac{1}{2} \beta_{2}{ }^{2}\left(\frac{S_{\frac{1}{1}}-S_{m 1}}{\sigma^{2}}+\frac{1}{\sigma_{2}{ }^{2}}\right)+\beta_{2}\left(\frac{S_{m 4}}{\sigma^{2}}\right)\right]} d \beta_{2}=\int_{-\infty}^{\infty} e^{\left[-\frac{1}{2} \beta_{2}{ }^{2} B_{2}+\beta_{2} A_{2}\right]} d \beta_{2}=\frac{e^{\frac{A_{3}{ }^{2}}{2 B_{2}}} \sqrt{2 \pi}}{\sqrt{B_{2}}} \\
g\left(m \mid \sigma^{-2}, \beta_{1}, \beta_{2}, Z\right) \propto e^{-\frac{1}{\sigma^{2}}\left(c+\frac{A+B}{2}\right)} \sigma^{-2\left(\frac{n}{2}+d-1\right)} \frac{e^{\frac{A_{1}{ }^{2}}{2 B_{1}} \sqrt{2 \pi}}}{\sqrt{B_{1}}} \frac{{\frac{A}{2 B_{2}}}^{2}}{\sqrt{B_{2}}}
\end{gathered}
$$

where $A, B, A_{1}, B_{1}, A_{2}$ and $B_{2}$ are given in equation (7).

\section{SOLUTION OF A NUMERICAL EXAMPLE:}

The two phase linear regression model is assumed as under:

$$
y_{t}=\left\{\begin{array}{lr}
3 x_{t}+\epsilon_{t}, & t=1,2,3,4 \\
3.5 x_{t}+\epsilon_{t}, & t=5,6, \ldots, 15
\end{array}\right.
$$

Here, the independently and identically distributed random errors which follow Normal Distribution $\mathbf{N}(\mathbf{0}, \mathbf{1})$ and are denoted by $\boldsymbol{\epsilon}_{\boldsymbol{t}}$. The generated observations are precisely given in TABLE $1 . \boldsymbol{\beta}_{\mathbf{1}}$ and $\boldsymbol{\beta}_{2}$ themselves were random observations from standard normal distribution with $\boldsymbol{\mu}_{\mathbf{1}}=\mathbf{0}$ and standard deviation $\sigma=1$ and precision $\frac{1}{\sigma^{2}}$ was from gamma distribution with (prior mean $\boldsymbol{\mu}_{3}=\mathbf{1}$ and $\emptyset^{2}=\mathbf{0 . 0 2}$ ). Also $\boldsymbol{c}=\mathbf{5 0}$ and $\boldsymbol{d}=\mathbf{5 0}$.

\section{TABLE 1}

DATA GENERATED FROM THE TPLR MODEL

\begin{tabular}{|c|c|c|c|c|c|c|c|c|c|c|c|c|c|c|}
\hline $\mathbf{T}$ & $\boldsymbol{x}_{\boldsymbol{t}}$ & $\boldsymbol{y}_{\boldsymbol{t}}$ & $\mathbf{T}$ & $\boldsymbol{x}_{\boldsymbol{t}}$ & $\boldsymbol{y}_{\boldsymbol{t}}$ & $\mathbf{t}$ & $\boldsymbol{x}_{\boldsymbol{t}}$ & $\boldsymbol{y}_{\boldsymbol{t}}$ & $\mathbf{T}$ & $\boldsymbol{x}_{\boldsymbol{t}}$ & $\boldsymbol{y}_{\boldsymbol{t}}$ & $\mathbf{t}$ & $\boldsymbol{x}_{\boldsymbol{t}}$ & $\boldsymbol{y}_{\boldsymbol{t}}$ \\
\hline $\mathbf{1}$ & 1.7 & 4.6 & 4 & 3.3 & 8.0 & 7 & 4.2 & 13.90 & 10 & 4.8 & 16.40 & 13 & 6.0 & 17.99 \\
\hline $\mathbf{2}$ & 2.2 & 6.4 & 5 & 3.9 & 13.75 & 8 & 4.5 & 14.18 & 11 & 5.1 & 16.90 & 14 & 6.2 & 18.92 \\
\hline $\mathbf{3}$ & 2.7 & 6.9 & 6 & 4.1 & 11.27 & 9 & 4.7 & 15.45 & 12 & 5.2 & 17.32 & 15 & 6.5 & 19.36 \\
\hline
\end{tabular}

From the above table, it is quite clear that a random sample of size $\boldsymbol{n}=\mathbf{1 0 0}$ is generated from $\boldsymbol{g}\left(\boldsymbol{m} \mid \boldsymbol{\sigma}^{-2}, \boldsymbol{Z}\right)$ using the Random Walk Metropolis - Hasting (RWM-H) algorithm for $\mathbf{5 0 0 0}$ times each. Further, we observe that the selected proposal is $\mathbf{U}(\mathbf{1}, \mathbf{1 4})$ which is same as the prior and it is symmetric around 7.5. Also, we note from the above table that the initial distribution is chosen as $\mathbf{U}(\mathbf{1}, \mathbf{1 4})$ since the target function is bounded. Moreover, the initial distribution is truncated and then we obtain the integer value of the Bayes Estimate of change point ' $\boldsymbol{m}$ ' as $\mathbf{4}$ when selected proposal is $\mathbf{U}(\mathbf{1}, \mathbf{1 4})$ and initial distribution is $\mathbf{U}(\mathbf{3}, \mathbf{1 4})$. The data given in TABLE 1 has its results in TABLE 2 when given values are taken as $\boldsymbol{\beta}_{1}=\mathbf{2}, \boldsymbol{\beta}_{2}=\mathbf{3}$ and $\boldsymbol{\sigma}^{2}=\mathbf{2}$.

TABLE 2

\section{BAYES ESTIMATES OF CHANGE POINT ' $m$ ' USING RWM-H ALGORITHM UNDER SQUARED ERROR LOSS FUNCTION}

\begin{tabular}{|l|l|l|l|}
\hline $\begin{array}{l}\text { Selected } \\
\text { Proposal }\end{array}$ & $\begin{array}{l}\text { Initial } \\
\text { Distribution }\end{array}$ & $\begin{array}{l}\text { Bayes Estimate of Change } \\
\text { Point ' } \boldsymbol{m} \text { ' }\end{array}$ & $\begin{array}{l}\text { Integer Value of Bayes } \\
\text { Estimate of Change } \\
\text { Point ' } \boldsymbol{m} \text { ' }\end{array}$ \\
\hline $\mathrm{U}(1,14)$ & $\mathrm{U}(1,14)$ & 1.66 & 2 \\
\hline $\mathrm{U}(1,14)$ & $\mathrm{U}(2,14)$ & 2.65 & 3 \\
\hline
\end{tabular}


PARIPEX - INDIAN JOURNAL OF RESEARCH | Volume - 10 | Issue - 06 | June - 2021 | PRINT ISSN No. 2250 - 1991 | DOI : $10.36106 /$ paripex

\begin{tabular}{|l|l|l|l|}
\hline $\mathrm{U}(1,14)$ & $\mathrm{U}(2,14)$ & 2.65 & 3 \\
\hline $\mathbf{U}(\mathbf{1 , 1 4})$ & $\mathbf{U}(\mathbf{3 , 1 4})$ & $\mathbf{3 . 5 5}$ & $\mathbf{4}$ \\
\hline
\end{tabular}

The results where the Bayes Estimates of ' $\boldsymbol{m}$ ' are computed using RWM-H algorithm for the different prior under consideration for the data given in TABLE 1 are shown in following TABLE 3.

TABLE 3

BAYES ESTIMATES OF CHANGE POINT ' $m$ ' USING RWM-H ALGORITHM UNDER SQUARED ERROR LOSS FUNCTION FOR DIFFERENT PRIOR UNDER CONSIDERATION

\begin{tabular}{|l|l|l|l|l|l|}
\hline Serial Number & $\boldsymbol{\mu}_{\mathbf{1}}$ & $\boldsymbol{\mu}_{\mathbf{2}}$ & $\boldsymbol{\sigma}_{\mathbf{1}}{ }^{2}$ & $\boldsymbol{\sigma}_{\mathbf{2}}{ }^{2}$ & $\begin{array}{l}\text { Bayes Estimate of Change Point ' } \boldsymbol{m} \text { ' } \\
\text { (Posterior Mean) }\end{array}$ \\
\hline 1 & 0 & 0 & 1000 & 12000 & 4 \\
\hline 2 & 2 & 3 & 1000 & 12000 & 4 \\
\hline 3 & 2 & 3 & 4 & 9 & 4 \\
\hline 4 & 2 & 3 & 1 & 9 & 4 \\
\hline 5 & 2 & 3 & 0.09 & 0.09 & 4 \\
\hline 6 & $\mathbf{2}$ & $\mathbf{3}$ & $\mathbf{0 . 0 1}$ & $\mathbf{0 . 0 9}$ & $\mathbf{4}$ \\
\hline 7 & 20 & 30 & 0.01 & 0.09 & 4 \\
\hline 8 & 0.2 & 0.3 & 0.01 & 0.09 & 4 \\
\hline
\end{tabular}

Here, we have used Gibbs Sampling Technique and MCMC algorithm for the different prior under consideration for the data given in TABLE $\mathbf{1}$ where we have computed the Bayes Estimates of $\boldsymbol{\beta}_{\mathbf{1}}$ and $\boldsymbol{\beta}_{\mathbf{2}}$ (when given value of $\boldsymbol{\beta}_{\mathbf{2}}=\mathbf{3}, \boldsymbol{m}=\mathbf{4}$ and $\boldsymbol{\sigma}^{\mathbf{2}}=\mathbf{2}$ ). The results are shown below in TABLE 4.

\section{TABLE 4}

BAYES ESTIMATES OF $\beta_{1}$ AND $\beta_{2}$ USING GIBBS SAMPLING TECHNIQUE AND MCMC ALGORITHM UNDER SQUARED ERROR LOSS FUNCTION FOR DIFFERENT PRIOR UNDER CONSIDERATION

\begin{tabular}{|c|c|c|c|c|c|c|c|c|c|c|}
\hline \multirow{2}{*}{$\begin{array}{l}\text { Serial } \\
\text { Number }\end{array}$} & \multirow[b]{2}{*}{$\mu_{1}$} & \multirow[b]{2}{*}{$\mu_{2}$} & \multirow{2}{*}{$\sigma_{1}^{2}$} & \multirow{2}{*}{$\sigma_{2}{ }^{2}$} & \multicolumn{3}{|c|}{ Bayes Estimates of } & \multicolumn{3}{|c|}{ Standard Deviation of Bayes Estimates of } \\
\hline & & & & & $\beta_{1}$ & $\beta_{2}$ & $\sigma^{2}$ & $\beta_{1}$ & $\beta_{2}$ & $\sigma^{2}$ \\
\hline 1 & 0 & 0 & 1000 & 12,000 & 2.573 & 3.168 & 2.348 & 0.237 & 0.112 & 0.022 \\
\hline 2 & 2 & 3 & 1000 & 12,000 & 2.573 & 3.168 & 2.348 & 0.237 & 0.112 & 0.022 \\
\hline 3 & 2 & 3 & 4 & 9 & 2.562 & 3.168 & 2.348 & 0.235 & 0.112 & 0.022 \\
\hline 4 & 2 & 3 & 1 & 9 & 2.568 & 3.168 & 2.348 & 0.236 & 0.112 & 0.022 \\
\hline 5 & 2 & 3 & 0.09 & 0.09 & 2.309 & 3.156 & 2.348 & 0.175 & 0.108 & 0.022 \\
\hline 6 & 2 & 3 & 0.01 & 0.09 & 2.065 & 3.156 & 2.348 & 0.081 & 0.108 & 0.022 \\
\hline 7 & 20 & 30 & 0.01 & 0.09 & 17.963 & 5.074 & 2.348 & 0.081 & 0.108 & 0.022 \\
\hline 8 & 0.2 & 0.3 & 0.01 & 0.09 & 0.475 & 2.964 & 2.348 & 0.081 & 0.108 & 0.022 \\
\hline
\end{tabular}

Here also we have used Gibbs Sampling Technique and MCMC algorithm for different prior under consideration for the data given in TABLE $\mathbf{1}$ and computed the Bayes Estimates of $\boldsymbol{\sigma}^{2}$ when given value of $\boldsymbol{\beta}_{\mathbf{1}}=\mathbf{2}, \boldsymbol{\beta}_{\mathbf{2}}=\mathbf{3}$ and $\boldsymbol{m}=\mathbf{4}$. The results are shown below in TABLE $\mathbf{5}$.

\section{TABLE 5}

BAYES ESTIMATES OF $\sigma^{2}$ USING GIBBS SAMPLING TECHNIQUE AND MCMC ALGORITHM UNDER SQUARED ERROR LOSS FUNCTION FOR DIFFERENT PRIOR UNDER CONSIDERATION

\begin{tabular}{|l|l|l|l|l|l|l|}
\hline Serial Number & $\boldsymbol{\mu}_{\mathbf{3}}$ & \multicolumn{1}{|c|}{$\boldsymbol{\varphi}^{\mathbf{2}}$} & $\boldsymbol{c}$ & $\boldsymbol{D}$ & $\begin{array}{c}\text { Bayes Estimates of } \\
\boldsymbol{\sigma}^{\mathbf{2}}\end{array}$ & $\begin{array}{l}\text { Standard Deviation of Bayes estimates of } \\
\boldsymbol{\sigma}^{\mathbf{2}}\end{array}$ \\
\hline $\mathbf{1}$ & $\mathbf{1}$ & $\mathbf{0 . 0 0 0 2}$ & $\mathbf{5 0 0 0}$ & $\mathbf{5 0 0 0}$ & $\mathbf{2 . 3 4}$ & $\mathbf{0 . 0 3 2}$ \\
\hline 2 & 0.1 & 0.002 & 5000 & 500 & 1.44 & 0.020 \\
\hline 3 & 0.5 & 0.002 & 1000 & 500 & 7.18 & 0.231 \\
\hline 4 & 5 & 0.002 & 100 & 500 & 67.72 & 6.727 \\
\hline 5 & 50 & 0.0002 & 100 & 5000 & 109.86 & 10.913 \\
\hline 6 & 5 & 0.0002 & 1000 & 5000 & 11.66 & 0.374 \\
\hline 7 & 2 & 0.0002 & 2500 & 5000 & 4.68 & 0.090 \\
\hline 8 & 1.67 & 0.0002 & 3000 & 5000 & 3.90 & 0.069 \\
\hline 9 & 0.6 & 0.000333 & 5000 & 3000 & 1.94 & 0.027 \\
\hline 10 & 0.5 & 0.0004 & 5000 & 2500 & 1.84 & 0.026 \\
\hline 11 & 0.1 & 0.002 & 5000 & 500 & 1.44 & 0.020 \\
\hline
\end{tabular}




\section{GRAPHS AND INTERPRETATIONS}

Here, GRAPH 1 shows the full conditional of $\boldsymbol{\beta}_{1}$ when a sample of size 10,000 was generated from $\boldsymbol{g}\left(\boldsymbol{\beta}_{1} \mid \boldsymbol{\beta}_{2}, \boldsymbol{\sigma}^{-2}, \boldsymbol{m}, \boldsymbol{Z}\right)$. Also, Gibbs Sampling with MCMC algorithm showed the results $\beta_{2}=3, \sigma^{2}=2, m=4$

Figure 1: Full Conditional of $\beta_{1}$

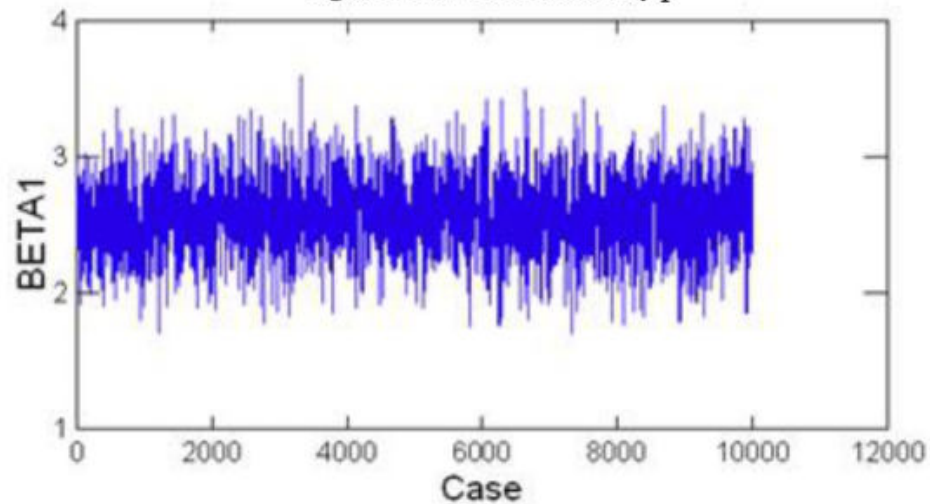

Here, GRAPH 2 shows the full conditional of $\boldsymbol{\beta}_{2}$ when a sample of size $\mathbf{1 0 , 0 0 0}$ was generated from $\boldsymbol{g}\left(\boldsymbol{\beta}_{\mathbf{2}} \mid \boldsymbol{\beta}_{\boldsymbol{1}}, \boldsymbol{\sigma}^{-\mathbf{2}}, \boldsymbol{m}, \boldsymbol{Z}\right)$. Also, Gibbs Sampling with MCMC algorithm showed the results $\beta_{1}=2, \sigma^{2}=2, m=4$

Figure 2: Full Conditional of $\beta_{2}$

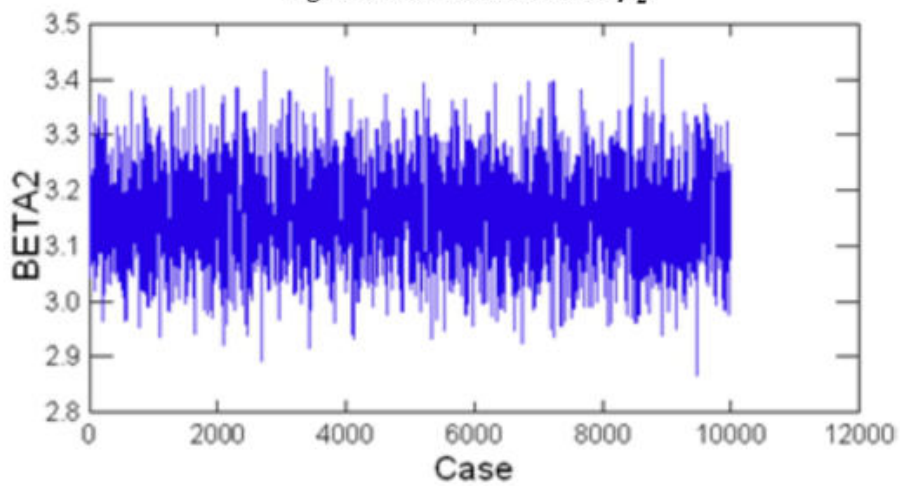

Here, GRAPH 3 shows the full conditional of $\boldsymbol{\sigma}^{-2}$ when a sample of size 10,000 was generated from $\boldsymbol{g}\left(\boldsymbol{\sigma}^{-2} \mid \boldsymbol{\beta}_{1}, \boldsymbol{\beta}_{2}, \boldsymbol{m}, \boldsymbol{Z}\right)$. Also, Gibbs Sampling with MCMC algorithm showed the results $\beta_{1}=2, \beta_{2}=3, m=4$

Figure 3: Full Conditional of $\sigma^{-2}$

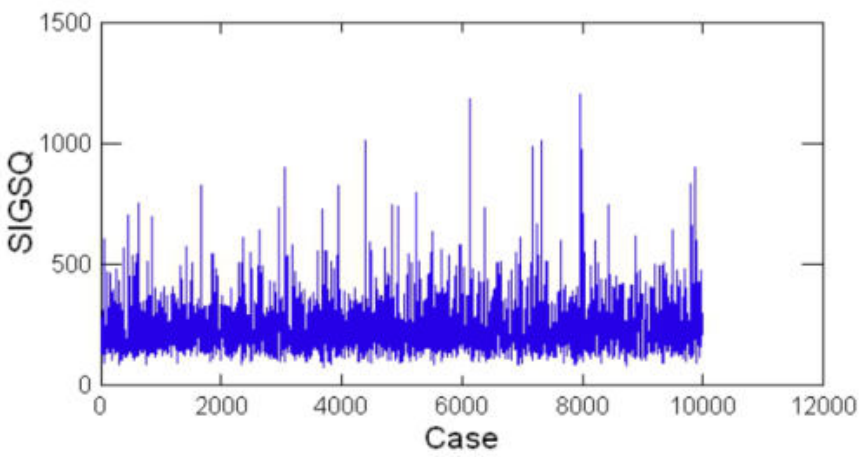

\section{REFERENCES}

[1] Zellner, Arnold (1971), "An Introduction to Bayesian Inference",JohnWiley and Sons, Inc., New York.

[2] Dyer, D. D. and Whisenand (1973). "Best Linear Unbiased estimator of the parameters of the Rayleigh distribution", IEEE Transactions on Reliability, R-22, 27-34 and $455-466$.

[3] Smith, A. F. M. (1980), “Change point problems:Approaches and Applications”, Bayesian Statistics (J.M. Bernardo, M.H.DeGroot,D.V.Lindley, and A. F.M. Smith, eds.), pp. 83-98, University Press,Valencia.

[4] L. D. Broemeling and H.Tsurumi (1987), "Econometrics and Structural Change", Marcel Dekker,NewYork, USA.

[5] Gelfand and Smith (1990), "Sampling-based approaches to calculating marginal densities",J.Am. Statist. Assoc. 85, pp. 398-409.

[6] A. K. Bansal and S. Chakravarty (1996), "Bayes estimation and detection of a change in prior distribution of the regression parameter", Bayesian Analysis in Statistics and Econometrics, Donald A. Berry and M. Kathryn, Eds.,pp. 257-266, Wiley-Interscience, New York, NY, USA.

[7] Jorge A. Achcar and Roseli A. Leandro (1998), "Use of Markov Chain Monte Carlo Methods in A Bayesian Analysis of the Block and Basu Bivariate Exponential Distribution",Ann.Inst.Statist.Math.,Vol.50. No.3,pp.403-416.

[8] S. K. Upadhyay and N. Vasistha (2000), "Bayes Inference In Life Testing and Reliability via Markov Chain Monte Carlo Simulation", Sankhya: The Indian Journal of Statistics. 2000, Vol.62. Series A,Pt.2,pp. 203-222

[9] Christian Robert and George Casella (2011), "A Short History of Markov Chain Monte Carlo: Subjective Recollection from Incomplete Data", Statistical Science 201 1 Vol.26. No.1,pp. 102-115 